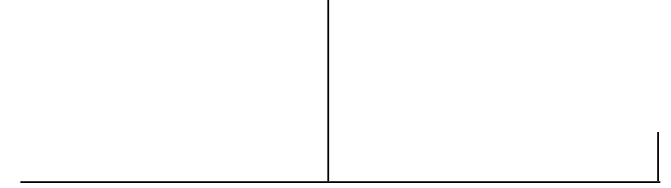

Rev. Latinoam. Psicopat. Fund., São Paulo, v. 11, n. 2, p. 195-207, junho 2008

\title{
Tempo e psicopatologia cultural das experiências traumáticas
}

Claudio Lyra Bastos

Relacionamos aqui dois aspectos fundamentais das intuições culturais sobre a passagem do tempo - a temporalidade cíclica e a contínua - com a psicopatologia e a terapêutica médica, psicológica e religiosa dos estados de estresse pós-traumáticos.

Nas concepções culturais cíclicas do tempo, vida e morte são indissociáveis do movimento eterno do cosmos. A severidade e a persistência do trauma mental não são diretamente proporcionais à magnitude da catástrofe, mas associadas às características imaginárias e aos papéis que representam na mente. A cultura moderna tende a produzir indivíduos preparados para um mundo altamente complexo, sob pressão constante em um ritmo frenético. Quase todos os eventos devem ser antecipados, planejados ou controlados e os traços anancásticos de personalidade são bem aceitos pelas sociedades modernas. Todavia, face a eventos catastróficos, imprevisíveis, quando nada resta a fazer, esses indivíduos metódicos e organizados podem apresentar fragilidade e desespero.

Nas comunidades tradicionais as vítimas parecem capazes de suportar níveis muito altos de agressão ou sofrimento - em situações traumáticas - sem mostrar sinais proporcionais de estresse mental. Enquanto que nos estratos superiores das comunidades modernas um ato de violência, como um assalto ou um estupro, pode ter conseqüências muito sérias e duradouras, na prática diária, nos 
hospitais públicos, encontramos pessoas que sofreram eventos potencialmente traumáticos sem qualquer dos esperados efeitos devastadores na sua vida mental. Os rituais dissociativos periódicos podem ter algum papel na sua resiliência.

Palavras-chave: Estresse pós-traumático, psicopatologia e cultura, temporalidade e medicina, dissociação

Este texto procura tecer uma rede de relações entre os conceitos culturais de temporalidade, alguns fatores patoplásticos na psicopatologia do trauma psíquico e os fenômenos dissociativos como instrumentos terapêuticos nas experiências traumáticas no Brasil.

Os únicos desastres naturais importantes no país são as inundações sazonais, raramente severas. A maior fonte de trauma mental é a atividade humana (acidentes de trânsito, violência urbana, guerras de quadrilhas etc.). Nas últimas décadas, o processo rápido e desorganizado de urbanização, assim como o explosivo crescimento do narcotráfico tornaram o país - cujos índices de homicídio e fatalidades de trânsito se encontram entre os maiores do mundo - muito violento. Em 2002, as causas violentas foram responsáveis por $70,67 \%$ de toda a mortalidade do sexo masculino entre 15 e 24 anos. Dezenas de milhares de jovens morrem ou ficam incapacitados a cada ano de forma violenta e repentina.

Apesar desse número imenso de vítimas, apenas uma pequena fração da população que busca ajuda psiquiátrica nos serviços públicos de saúde apresenta sintomas compatíveis com o Transtorno de Estresse Pós-Traumático (TEPT). A par disso, desde o trabalho de Mari (1983), comparando o número de ambulatórios psiquiátricos com o número de centros espíritas, sabemos da enorme insuficiência ou desproporção dos primeiros. A situação hoje não é muito diferente. Se a maioria dessas pessoas realmente não precisa de qualquer ajuda ou se encontram o que procuram em outros lugares, podemos apenas especular. Em ambas as alternativas, porém, fatores culturais representam um papel significativo. 
Relacionamos dois aspectos das concepções culturais e intuitivas da passagem do tempo - a temporalidade cíclica e a contínua - com a psicopatologia clínica do chamado transtorno de estresse pós-traumático (TEPT) no Brasil (Bastos, 1996).

1) Concepções cíclicas do tempo mostram forte dependência dos ciclos da natureza e do tempo cósmico, como as estações do ano e os movimentos dos corpos celestiais. A cura é praticada em rituais periódicos e terapias cíclicas, geralmente envolvendo técnicas dissociativas, como a possessão por espíritos.

2) Concepções temporais seqüenciais ou contínuas pressupõem um tempo humano, independente dos ciclos naturais, indicando autodeterminação, autonomia e individualismo. ${ }^{1} \mathrm{Na}$ medicina moderna, as terapias passam a ter metas individuais, sendo planejadas e programadas de forma contínua, seqüencial.

\section{Cultura e psicopatologia}

As concepções da passagem do tempo traçam os caminhos pelos quais os diferentes grupos religiosos tendem a ver os eventos traumáticos. Duas tendências básicas podem ser vistas em qualquer um deles, mesmo que sob formas ambíguas como as que encontramos no Brasil (Bastos, 2005, 2006):

1) Em ambientes culturais aonde o tempo cíclico predomina, a maior parte das pessoas ocupa-se apenas com o tempo presente. Passado e futuro não são levados muito a sério. Há uma tendência à temporalização do espaço: distâncias podem ser medidas em minutos, horas ou dias. Perspectivas fatalistas são comuns e a esperança repousa nas crenças de ajuda ou salvação externa. A improvisação tende a ser grande, mas a prevenção é quase ausente. Causas externas são sempre atribuídas aos eventos catastróficos ou traumáticos, que tendem a ser encarados como inevitáveis expressões da vontade de Deus. Tragédias são vistas como partes da vida e o sofrimento pode ser interpretado como uma forma de penitência. Falhas dos mecanismos psicológicos comuns de defesa conduzem a reações depressivas com características hipocondríacas; o "mau-olhado" ou "olho gordo" e a feitiçcom freqüencia.

1. A doutrina protestante (calvinista) da predestinação não contradiz as tendências internalizantes da cultura tradicional americana, à diferença das sociedades que tendem ao fatalismo e à externalidade. Os calvinistas buscam o controle total das suas vidas para que se confirme o fato inexorável de que eles são mesmo os predestinados à salvação. 
2) Nos meios em que o tempo contínuo predomina, as pessoas tendem a ser mais individualistas e comprometidas com o controle interno e externo, contando com a previsibilidade de quase tudo. Passado e futuro passam a ter enorme importância. Há tendência à espacialização do tempo: horas, dias e meses são esquematizados em mapas, cartas e diagramas, sendo tratados como distâncias. Eventos traumáticos rompem a ordem seqüencial e a estrutura da vida, balançando a fé das pessoas na previsibilidade. O sofrimento tende a ser relacionado à causalidade interna, originando sentimentos de fracasso e atitudes auto-incriminadoras. Reações depressivas mostram profundos traços de culpa ou vergonha.

\section{A religião como agente terapêutico}

A religião é, de longe, o mais importante provedor de saúde mental no Brasil. Acolhimento, alívio, apoio e compreensão empática são elementos comuns a qualquer abordagem terapêutica eficaz. Além disso, também se inclui aí uma interpretação dos fatos, sentimentos e eventos de acordo com um arcabouço mitológico específico (Lévi-Strauss, 1976). Ela trata os problemas psiquiátricos (especialmente os quadros chamados neuróticos) de três formas genéricas principais, que não se distinguem essencialmente dos próprios traços ditos "neuróticos": ${ }^{2}$

1) Rituais anancásticos de controle (do grego ananke: necessidade): liturgias e compromissos obsessivo-compulsivos, como os rituais cognitivo-comportamentais e de empowerment. A maior parte dos instrumentos de cura nas modalidades modernas de psicoterapia tem fortes características internalizantes e de controle, incluindo aí as terapias cognitivas e as psicanalíticas. O individualismo moderno se acha fortemente vinculado ao autocontrole e à autodisciplina. Terapeutas cognitivos tendem a exercer esse controle de forma mais ou menos clara e explícita, enquanto que terapeutas de orientação psicanalítica tendem a fazê-lo de forma mais sutil, por meio da transferência.

2) Rituais de tabu: mecanismos evitativos, fóbicos. Localizando o mal, torna-se possível evitá-lo. Assim como muçulmanos e judeus não podem comer carne de

2. O que distingue o traço neurótico do elemento terapêutico não é nenhuma característica intrínseca, mas sim o seu aspecto de desequilíbrio, de inadequação, de sofrimento (pathos). O equilíbrio dinâmico entre mecanismos controladores, dissociativos, evitativos, e mesmo paranóides, é o que faz funcionar as personalidades normais e a vida social. Cada ambiente cultural tende a valorizar alguns aspectos desse equilíbrio enquanto patologiza outros. 
porco, e budistas devem se abster de carne, seguidores dos preceitos da Boa Saúde moderna devem evitar açúcar, sal, carnes vermelhas e colesterol. Preconiza-se que muitos problemas emocionais e relações conflituosas podem ser neutralizados com o afastamento dos maus alimentos e dos maus ambientes, mantendo o corpo e a mente livres de maus hábitos, más palavras, maus germes, maus produtos químicos, más gorduras etc. Retiros, spas e rituais purificatórios limpam o corpo e a alma do estresse e da poluição física e mental.

3) Rituais dissociativos, de transe e possessão: mecanismos de estreitamento, bloqueio e focalização da atenção, assim como aumento da externalização, da sensibilidade coletiva e da sugestibilidade, proporcionando a criação de novas perspectivas e novos espaços de autoconsciência. São vistos como patológicos no DSM-IV, mas mostram-se como elementos essencialmente terapêuticos em muitas culturas (ver Castillo, 1997). Na própria sociedade americana, os cultos pentecostais mostram importantes aspectos dissociativos; muitas seitas protestantes se iniciaram dessa forma para depois evoluírem e se consolidarem por meio de ritos predominantemente controladores. A preferência por este ou aquele ritual é guiada por princípios ideológicos e mitológicos (no sentido antropológico) de cada religião, época ou grupo social. ${ }^{3}$

Todas as práticas de cura psíquica - de natureza religiosa ou não - tendem a proporcionar a cura através do significado dado à experiência traumática (de acordo com a sua respectiva mitologia). Cultos modernos tendem a fazê-lo especialmente pelo controle e isolamento do mal. Cultos tradicionais também usam o isolamento, mas dão forte preferência à dissociação como instrumento terapêutico - especialmente nos transtornos traumáticos - para promover mudanças na personalidade e criar as possibilidades de uma nova vida.

Nestes últimos casos, as terapias não se acham orientadas seqüencialmente quanto ao tempo: não há roteiro, planejamento estratégico ou meta a ser alcançada; devem ser repetidas ou renovadas sempre. Rituais de morte periódica e renascimento são essenciais às Weltanschauungen de tempo cíclico. Fenômenos dissociativos não funcionam bem em programas evolutivos, nos quais as coisas

3. Uma reportagem do Der Spiegel sobre o crescimento das igrejas evangélicas no Brasil diz: “Os fiéis não fazem objeção à cobiça de seus líderes: a riqueza em si não é considerada um pecado, mas sim desejável. Nas comunidades pentecostais, qualquer um pode se tornar um pastor... No final dos anos 80, um estudo altamente divulgado do antropólogo americano Sheldon Annis concluiu que as comunidades protestantes são comercialmente mais bem-sucedidas que as comunidades católicas. Annis comparou a produtividade das operações de tecelagem em duas aldeias na Guatemala. Suas conclusões apontaram que, em comparação aos católicos, os pentecostais adotaram técnicas modernas de produção mais rapidamente, eram mais eficientes e mais preocupados em progredir."

Rev. Latinoam. Psicopat. Fund., São Paulo, v. 11, n. 1, p. 195-207, junho 2008 
devem melhorar sempre até que se tornem completamente previsíveis e sob controle. Suas vias terapêuticas tendem a ser periódicas, cíclicas e repetitivas, incluindo os aspectos simultâneos e ambíguos da personalidade e de sua realidade.

\section{Dissociação como instrumento terapêutico}

Pierre Janet $(1889,1923)$, entre outros, já havia destacado a importância dos fenômenos dissociativos como reações defensivas básicas de defesa da mente contra o trauma. De acordo com ele, nos eventos traumáticos accompagnés d'une violente émotion poderia haver um retraimento da consciência (rétrécissement de conscience) no qual as lembranças traumáticas (souvenirs traumatiques) poderiam aparecer automaticamente (como um automatisme psychologique).

A importância dos métodos dissociativos, como o transe e a hipnose, na terapêutica já foi extensivamente demonstrada por inúmeros autores, de todas as vertentes teóricas (reflexologistas, psicanalistas etc.), desde o século XVIII. No entanto, os modelos psicoterapêuticos modernos tendem - etnocentricamente a se basear quase que exclusivamente em instrumentos de controle e a não considerar os fenômenos dissociativos senão como eventos essencialmente patológicos (Thakker, 1998), desconsiderando os seus papéis terapêuticos, evidentes em muitos outros ambientes culturais.

Nas últimas décadas, tanto os terapeutas de orientação psicanalítica como os de orientação cognitivo-comportamental têm encarado com desprezo qualquer forma de técnica dissociativa na terapêutica como arcaica, primitiva e inconfiável, como a hipnose e a feitiçaria.

De acordo com a nossa própria experiência clínica e antropológica,

1) No TEPT, como nas assim chamadas neuroses traumáticas, causadas por perdas ou eventos traumáticos, técnicas dissociativas mostram-se bastante eficazes. Além de simplesmente proporcionar a catarse, as experiências dissociativas podem facilitar a criação de identidades auxiliares que reassegurem o sentido para a vida do paciente.

2) A fragilidade face ao trauma e ao caos tende a ser maximizada quando a rigidez e o controle do presente se estendem ao futuro e à sua programação. Numa perspectiva seqüencial, o trauma tende a destruir o senso de futuro.

3) Por outro lado, quanto mais difícil é a mudança de uma Weltanschauung tradicionalmente fatalista, repetitiva e cíclica para uma outra, individualizante, burocratizante, planejada e sucessiva, menor a adaptação à pressão constante da vida moderna, e menor também a resiliência face aos eventos catastróficos (inadaptação e exclusão social são fatores importantes no TEPT - ver Marsella, 1996). 
A severidade e a persistência do trauma mental não estão diretamente relacionadas com a magnitude da catástrofe ou mesmo da experiência traumática, mas com a forma com que ocupam a mente e com os papéis que nela representam. Parece haver duas maneiras básicas de encarar o desastre, ilustradas na fábula de Esopo "A árvore e o caniço": a árvore é grande e rígida, porém não consegue resistir a uma tempestade realmente forte, que a derruba; já o humilde caniço se dobra todo frente ao vento, mas se vê de pé novamente quando a tormenta passa.

A cultura moderna tende a produzir indivíduos preparados para um mundo altamente complexo, correndo num ritmo frenético sob constante pressão. Quase todos os eventos devem ser previstos, planejados ou controlados, e os traços obsessivos de personalidade se vêem altamente favorecidos. Nos eventos catastróficos e imprevisíveis, porém, quando nada pode ser feito, muitas pessoas metódicas e de agenda detalhada podem mostrar descontrole, fragilidade e desespero. Nesse caso, o único sentido possível que se pode encontrar no desastre é encarálo como um desafio individual que só os bravos e fortes podem vencer. A única forma pela qual os mecanismos de controle podem lidar com o trauma é objetificando-o como uma coisa ou uma doença que causa dor e deve ser interrompida ou eliminada.

Porém, numa perspectiva tradicional, o trauma pode ser visto de forma completamente diferente, como uma eventualidade da existência humana, uma experiência que é parte essencial da vida (Fodor, 2001). Comunidades tradicionais parecem capazes de suportar níveis extremamente elevados de agressão ou sofrimento - nas situações traumáticas e catastróficas - sem mostrar sinais proporcionais de estresse mental. Rituais dissociativos periódicos podem exercer algum papel em sua resiliência.

Enquanto nos estratos sociais mais elevados das comunidades modernas um ato de violência, como um assalto ou um estupro, pode ter conseqüências sérias e duradouras, na prática diária dos hospitais públicos observamos eventos potencialmente traumáticos sem quaisquer dos efeitos devastadores sobre a vida mental das vítimas que tendemos a esperar. É possível que o exacerbado senso de responsabilidade pelo seu próprio destino, traço característico da percepção individualista e seqüencial do tempo, tenha algo a ver com isso. Internalizando as causas de todos os eventos, a sociedade moderna tende a produzir um forte compromisso entre os indivíduos e os seus próprios infortúnios. Por outro lado, onde a idéia circular do tempo prevalece - como na vida rural e em alguns estratos inferiores das sociedades modernas - apenas a Vontade de Deus e o inexorável Destino são responsáveis pelos desastres, absolvendo as pessoas de qualquer responsabilidade ou culpa. 
Religião e rituais de cura no Brasil têm muitas raízes culturais entrelaçadas: origens históricas portuguesas, católicas, influenciadas pelo espiritismo kardecista, pela maçonaria e pelo positivismo, na classe média, e rituais afro-brasileiros nas classes inferiores. Nos últimos vinte anos o protestantismo - especialmente os cultos pentecostais - espalhou-se enormemente por todo o país. ${ }^{4}$

Mecanismos dissociativos de defesa são muito comuns e não são percebidos como patológicos pela população. Nos cultos tradicionais afro-brasileiros, são vistos muito mais como tratamentos do que como sintomas ou doenças. Não existem casos de "personalidades múltiplas" porque milhões de pessoas se vêem rotineiramente possuídas por entidades sobrenaturais nos terreiros, em rituais circulares, durante toda a sua existência. Temos aqui um ritual periódico de descarrego, em que todas as más influências externas são eliminadas. Mesmo nos cultos protestantes - especialmente pentecostais - os estados dissociativos e o vocabulário afro-brasileiro ("encosto", "exu”, "descarrego") são mantidos como elementos fundamentais. Para os crentes, o modelo terapêutico baseado na seqüência doença-tratamento-cura parece não ter qualquer sentido, assim como não tem a prevenção, a não ser que ritualizada de forma periódica.

Disse um pai-de-santo: "O sentido do tempo na Umbanda é diferente. Não é como um curso que lhe dá um diploma no fim. Na Umbanda não existe um tempo como esse; cada um tem o seu próprio tempo." Isto é precisamente o que o antropólogo Edward Hall chamou de Tempo Policrônico, por oposição ao Tempo Monocrônico da vida moderna, no qual os calendários e relógios determinam o único tempo válido.

Nas reações de estresse traumático devido a perdas pessoais insuportáveis - como a morte de um filho - a umbanda e o espiritismo kardecista se mostram especialmente disponíveis a serem procurados como apoio, uma vez que lidam com as almas dos mortos. Suas abordagens tendem a ser intensamente fatalistas uma vez que entendem que todos os eventos presentes estão relacionados ao passado, em ciclos eternos.

Ouvir vozes, ver imagens e receber mensagens do além - ou mesmo perceber odores peculiares - são fenômenos dissociativos que podem ser facilmente induzidos e podem ter um enorme potencial redutor do sofrimento. Esses

4. O meteórico crescimento das comunidades evangélicas no Brasil e por toda a América Latina - às expensas dos cultos tradicionais - certamente tem algo a ver com essa busca por caminhos mais individualizantes. No entanto, essa expansão se dá quase que exclusivamente por meio dos cultos pentecostais, cujos pontos de interseção com os ritos tradicionais residem exatamente nas suas práticas dissociativas, e não nos seus fundamentos ideológicos e mitológi$\cos$. 
instrumentos terapêuticos podem trazer conforto e sentido para pessoas desesperadas. Parece haver um limiar de perdas para além do qual não é mais possível o retorno. Já não há mais futuro e os sintomas depressivos aparecem.

Durante a Segunda Guerra, psiquiatras britânicos usaram muito - e com bastante sucesso - técnicas hipnóticas e catárticas para tratar as assim chamadas "neuroses de guerra" (Sargant, 1942; Spiegel, 1987). Hoje, os psiquiatras tendem a depender quase que exclusivamente das drogas psicotrópicas e das abordagens cognitivas, que não parecem ser tão eficazes nos pacientes com TEPT, exceto quando vinculadas a técnicas catárticas.

Onde as concepções de tempo contínuo predominam, o senso de futuro é essencial, apontando para uma direção, uma meta. Passado, presente e futuro estão ligados em uma seqüência inseparável, como uma cadeia que, uma vez quebrada, se torna inútil. Em ambientes onde predomina uma cultura de tempo circular, o futuro pode não ser tão importante, já que o porvir está ligado ao passado e a ele sempre se retorna; a capacidade de suportar as perdas pode ser enorme.

Nós, brasileiros, tendemos a uma atitude extremamente ambígua quanto à passagem do tempo. Há um forte anseio pelo futuro, mas sem qualquer continuidade, uma vez que não temos passado. Tudo está sempre recomeçando do zero, tudo precisa sempre ser reerguido do chão, incontáveis vezes. A atitude quanto à religião reflete essa duplicidade. De acordo com DaMatta (1990), o dilema brasileiro é único em sua ambigüidade eterna, "escolhendo não escolher" entre o candomblé, o catolicismo, o kardecismo, a umbanda e mesmo o pentecostalismo (em princípio, oposto a todos os outros por sua maior rigidez e intolerância). Até que ponto essa ambigüidade tende à estagnação ou tem um valor adaptativo só o tempo nos dirá.

Desta estranha maneira, no Brasil, ambas as perspectivas do tempo interagem de uma forma complexa. Como diz DaMatta (1990): “... há um sistema, mas o que predomina não é o todo, mas as partes” (p. 44). Comparando eventos públicos como o Sete de Setembro e o Carnaval, o autor destaca que o primeiro corresponde a “... uma temporalidade registrada, empírica, que tem um início documentado e que faz parte de um conjunto de momentos críticos da vida brasileira os quais são vistos como encadeados" (ibid.). Essa temporalidade é claramente “... marcada pelo sentido de progresso, evolução e sobretudo, não-repetição" (ibid.). No segundo caso, porém, “... o Carnaval se situa numa escala cronológica cíclica, que independe de datas fixas" (ibid.). Esta cronologia tem uma natureza divina, cósmica, relacionada a valores universais e transcendentes.

Aqui descrevemos um caso clínico de trauma psíquico em que ocorre uma perda pessoal com sofrimento profundo, mas sem culpa. O paciente era um homem de 58 anos que havia perdido o seu filho de forma muito violenta. Ficou deprimido, extremamente ansioso, procurando ajuda de todas as fontes: terapias médicas, 
psicológicas, alternativas e religiosas ao mesmo tempo. Suas melhoras foram muito modestas e a maior parte dos sintomas persistiu ao longo de muitos meses.

Foi quando então, em uma das muitas casas de culto espírita que freqüentava, recebeu mensagens mentais de entidades espirituais que lhe disseram que seu filho estava bem, entre pessoas boas do mundo sobrenatural. Acreditou na mensagem e um dia, quando sentiu o aroma do perfume favorito de seu filho, no quarto do rapaz, finalmente teve a certeza de que tudo estava bem, e o seu sofrimento cessou. Os sintomas depressivos remitiram assim que ele entendeu que deveria viver sua vida sem amargura e que um dia voltaria a estar com seu filho. Ocorreu aqui uma recuperação posterior do sentido de futuro, desaparecido com o esvaziamento do presente. A busca da ajuda externa proporcionou a cura com dissociação.

\section{Conclusão}

Hoje, as técnicas psicoterápicas dissociativas têm o seu uso restrito aos terapeutas religiosos ou alternativos, sendo em geral ignoradas ou desprezadas pela psiquiatria e pela psicologia acadêmicas, apesar de constituírem a base históricoclínica de ambas as terapias psicanalítica e comportamental. Na visão modernizante da realidade, apenas os instrumentos terapêuticos que lidam com o controle têm valor científico.

No entanto, nas situações catastróficas, as possibilidades de controle freqüentemente desaparecem e as formas dissociativas de reequilíbrio podem ser os instrumentos terapêuticos mais adequados face ao caos, inclusive em pacientes de características controladoras, anancásticas.

Nos contextos culturais apropriados, especialmente aqueles em que as concepções cíclicas do tempo predominam, terapias baseadas na dissociação se mostram claramente eficazes no TEPT, permitindo a restauração do auto-respeito e proporcionando sentido a um novo período da vida. Uma vez que relativamente poucos pacientes procuram ajuda das instituições públicas de saúde ou instituições acadêmicas podemos concluir que deveria haver um novo olhar para esses instrumentos de cura.

\section{Referências}

Bastos, C.L. O tempo, as representações sociais de doença e a psicoterapia. Informação Psiquiátrica, Rio de Janeiro, v. 15, n. 4, p. 139-144, 1996. 
. Temporalidade e cultura. Revista Latinoamericana de Psicopatologia Fundamental, São Paulo, v. 8, n. 4, p. 738-753, dez.2005.

. Uma investigação sobre a temporalidade e a medicina. Revista Latinoamericana de Psicopatologia Fundamental, São Paulo, v. 9, n. 1, p. 80-113, mar.2006.

Castillo, R.J. Meanings of Madness. New York: Brooks/Cole, 1997.

DAMatTA, R. Carnavais, malandros e heróis. 5. ed. Rio de Janeiro: Guanabara Koogan, 1990.

FoDOR, L. The Reality of the Unmediated: Traumatic and Mystical Experience. eSharp, Glasgow, issue 7, Spring 2006 (Faith, Belief and Community).

Hall, E.T. The Dance of Life: The Other Dimension of Time. New York: Anchor, 1984.

JANET, P. (1889). L'Automatisme Psychologique. Paris: Odile Jacob, 1998.

. La Médecine Psychologique. Paris: Ernest Flammarion, 1923.

LÉVI-STRAuSs, C. Structural Anthropology. (Anthropologie Structurale Deux). New York: Basic Books, 1976. v. II.

Mari, J.J. Psychiatric care in Brazil. In: Brown, S. (ed.). Psychiatry in Developing Countries. London: Gaskell, 1983.

Marsella, A.J.; Friedman, M.J.; Gerrity, E.T.; Scurfield, R.M. (eds.). Ethnocultural Aspects of Posttraumatic Stress Disorder. Washington, D.C: A.P.A., 1996.

Mezzich, J.E.; Kleinman, A.; Fabrega, H.; Parron, D.L. Culture \& Psychiatric Diagnosis. Washington, D.C.: American Psychiatric Press, 1996.

Sow, I. Psychiatrie Dynamique Africaine. Paris: Payot, 1977.

Spiegel, H.; Spiegel, D. Trance and Treatment: Clinical Uses of Hypnosis. New York: Basic Books, 1987.

ThaKkER, J; WARD, T. Culture and classification: the cross-cultural application of the DSM-IV. Clin Psychol Rev, v. 18, n. 5, p. 501-29, Aug.1998.

\section{Resumos}

(Tiempo y psicopatología cultural de las experiencias traumáticas)

Relacionamos aqui dos aspectos fundamentales de las intuiciones culturales sobre el pasaje del tiempo - la temporalidad cíclica y la continua - con la psicopatología y la terapéutica médica, psicológica y religiosa de los estados de estrés post-traumáticos.

En las concepciones culturales cíclicas del tiempo, vida y muerte son indisociables del movimiento eterno del cosmos. La severidad y la persistencia del trauma mental no son directamente proporcionales a la magnitud de la catástrofe, sino 
asociadas a características imaginarias y papeles que representan en la mente. La cultura moderna tiende a producir individuos preparados para un mundo altamente complejo, bajo presión constante y en ritmo frenético. Casi todos los eventos deben ser anticipados, planeados o controlados y los rasgos anancasticos de personalidad son bien aceptos por las sociedades modernas. Además, ante eventos catastróficos, imprevisibles, cuando nada queda por hacer, estas personas controladas y rígidas pueden presentar fragilidad y desespero.

En las comunidades tradicionales las victimas parecen capaces de soportar niveles muy altos de agresión o sufrimiento - en situaciones traumáticas - sin presentar señales proporcionales de estrés mental. En tanto que en los estratos superiores de las comunidades modernas un acto de violencia como un asalto o una violación puede tener consecuencias muy serias y duraderas, en la práctica diaria en hospitales públicos encontramos personas que han sufrido eventos potencialmente traumáticos sin cualquiera de los esperados efectos devastadores en su vida mental. Los rituales disociativos periódicos pueden tener algún papel en su resiliencia.

Palabras clave: Estrés post-traumatico, psicopatología y cultura, temporalidad y medicina, disociación

(Temps et psychopathologie culturelle des expériences traumatiques)

Ce texte cherche à établir le rapport entre deux formes essentielles de conceptions intuitives culturelles sur le passage du temps - la temporalité cyclique et la continue - avec la psychopathologie et la thérapeutique de l'état de stress post-traumatique au Brésil. Dans les conceptions culturelles du temps de tendance cyclique, la vie et la mort sont indissociables dans le mouvement éternel du cosmos. La sévérité et la persistance du trauma mental ne sont pas directement proportionnelles à la magnitude de la catastrophe, mais associées à ses caractéristiques imaginaires et aux rôles qu'elles représentent dans l'esprit. La culture moderne tend à produire des individus préparés pour un monde très complexe, sous pression permanente et au rythme frénétique. Presque tous les événements doivent être anticipés, planifiés ou maîtrisés et les traits anankastiques de la personnalité sont bien acceptés par les sociétés modernes. Pourtant, face à des événements catastrophiques, imprévisibles, quand plus rien n'est à faire, ces personnes contrôlées et rigides peuvent présenter de la fragilité et du désespoir.

Dans les communautés traditionnelles, les victimes semblent capables de supporter de très hauts niveaux d'agression ou de souffrance - dans des situations traumatiques - sans présenter des signes proportionnels de stress mental, alors que dans les couches supérieurs des communautés modernes, un acte de violence, comme un hold-up ou un viol, peut avoir des conséquences très sérieuses et durables. Dans les hôpitaux publics, nous rencontrons quotidiennement des victimes d'événements potentiellement traumatiques sans qu'aucun des effets dévastateurs attendus se manifeste sur leur vie 
mentale. Les rituels dissociatifs périodiques pourraient jouer un rôle dans leur récupération.

Mots clés: Stress post-traumatique, psychopatologie et culture, temporalité et médecine, dissociation

(Time and the cultural psychopathology of traumatic experiences)

The present article relates two fundamental aspects of intuitive cultural conceptions of the passage of time - namely, cyclical and continuous - to the clinical psychopathology of post-traumatic stress in Brazil. People with predominantly cyclical cultural perceptions of time tend to see life and death as part of an eternal movement. For them, the severity and persistence of mental trauma are not directly related to the magnitude of a given catastrophe or traumatic experience. Rather, they are associated with characteristics of the mind and the roles they represent in the mind. Modern culture tends to produce individuals prepared for a highly complex world where nearly everyone is subject to constant pressure and a frantic pace of life. Almost all events must be anticipated, planned or controlled, and obsessive traits tend to be quite welcome in such a society. But when unpredictable catastrophic moments come up, when nothing can be done, such methodical persons may show frailty and great frustration.

Traditional communities seem able to bear extremely high levels of aggression or suffering - in traumatic and catastrophic situations - without showing proportional signs of mental stress. Whereas in upper social layers of modern communities acts of violence, such as muggings or rape, can have serious and long-lasting consequences on the victims, in everyday public hospital practice we come across victims of such seemingly traumatic events who do not show any of the usual devastating effects on their mental life. Periodic dissociative rituals may have a role in the resilience of these patients.

Key words: Post-traumatic stress, psychopatology and culture, temporality and medicine, dissociation

Versão inicial recebida em abril de 2007 Versão aprovada para publicação em março de 2008

\section{Cláudio lyra Bastos}

Médico Psiquiatra da Universidade Federal Fluminense (Niterói, RJ, Brasil); diretor do Instituto Fluminense de Saúde Mental (Niterói, RJ, Brasil)

Rua Guilherme Briggs, 51 - São Domingos

24210-175 Niterói, RJ

e-mail: clbastos56@uol.com.br

Rev. Latinoam. Psicopat. Fund., São Paulo, v. 11, n. 1, p. 195-207, junho 2008 